\title{
Sliding mode control of a dc-dc dual active bridge using the generalized space-state averaging description
}

\author{
Arnau Dòria-Cerezo ${ }^{1}$, Federico M. Serra ${ }^{2}$, Domingo Biel $^{3}$ and Robert Griñó ${ }^{4}$
}

\begin{abstract}
This paper presents a sliding mode control strategy for a dc-dc dual active bridge converter. The controller is based on a truncated model obtained using the generalized state space averaging method that transforms the mixed dcac dynamics of the converter into a regulation problem. The proposed controller, that uses a dynamic extension to overcome the structural problem of the non-affine control input, provides good results in terms of performance and robustness. Numerical simulations are included to validate the proposed modelling methodology and the control design.
\end{abstract}

\section{INTRODUCTION}

The dual active bridge (DAB) is an isolated dc-dc converter made of two active bridges interconnected with a high-frequency transformer. This converter can have a threephase [1] or single-phase topology [2] and the main features are high power density, bidirectional power flow, galvanic isolation, and the possibility of soft switching [3]. Due to the mentioned characteristics, the DAB converter is used in several applications such as microgrids [4], [5], electric vehicles [6], energy storage systems [7], solid-state transformer in medium-voltage and low-voltage distribution networks [8] among others.

The DAB converter is a non-linear dynamical system that mixes two dc stages (input and output) with an ac stage in between due to the magnetic transformer. This makes not possible to adopt the equivalent circuit model for designing control strategies and requires of the modification the model. The simplest way is to obtain first order nonlinear dynamics based on the power flow, see [9] for a detailed discussion on this behavioural modelling. Many papers propose a linearization around the equilibrium point to obtain a control-oriented model. Then, linear control techniques can be applied such as PI controllers [10], phase compensators [11], linear observers [12], or discrete-time linear controllers [13]. Some other papers propose nonlinear control strategies, including passivity-based techniques [14], [15], the feedback

\footnotetext{
*This work was supported in part by the Government of Spain through the Agencia Estatal de Investigación under Project DPI2017-85404-P and in part by the Generalitat de Catalunya under Project 2017 SGR 872.

${ }^{1}$ Arnau Dòria-Cerezo is with the Dept. of Electrical Engineering and Inst. of Industrial and Control Engineering, Universitat Politècnica de Catalunya, Barcelona, Spain. arnau. doria@upc.edu

${ }^{2}$ Federico M. Serra is with the Laboratorio de Control Automático (LCA), Universidad Nacional de San Luis and CONICET, 5730, Villa Mercedes, San Luis, Argentina. fmserra@unsl . edu. ar

${ }^{3}$ Domingo Biel is with the Dept. of Electronic Engineering and Inst. of Industrial and Control Engineering, Universitat Politècnica de Catalunya, Barcelona, Spain. domingo.biel@upc.edu .

${ }^{4}$ Robert Griñó is with the Dept. of Automatic Control and Inst. of Industrial and Control Engineering, Universitat Politècnica de Catalunya, Barcelona, Spain roberto.grino@upc.edu
}

linearization approach [16], or a double integral sliding mode control [17].

Alternatively, the generalized state space averaging (GSSA) methodology was proposed for a DAB converter in [18] and, later, extended in [19]. The GSSA expansion was firstly presented in [20] with the aim of capturing the fine detail of the state evolution by considering a full Fourier series. See examples of applications in modelling [21] and control [22], [23] of full-bridge rectifiers, and a general overview of the GSSA technique applied to power converters in [24]. With respect to the behavioural modelling, the advantage of using the GSSA approximation is that the resulting model provides more physical insight, but still nonlinear. Several linear controllers have been designed using the GSSA approximation of a DAB converter: PI-regulators [8], [25], optimal Linear Quadratic Gaussian control [26] or $H_{\infty}$ control [27]. Nonlinear control examples applied to a GSSA model include passivity-based controllers [14], [15]. The aforementioned control techniques usually rely on the knowledge of many parameter and variables that are used for the linearization procedure or appear in the feedback control law.

The contribution of this paper is a sliding mode controller based on the GSSA model of DAB converter. The main advantages with respect to the previous controllers is that the response dynamics can be freely designed through the switching manifold independently of the load and regulation parameters. Compared with [17], the proposed control algorithm does not require the exact values of the DAB converter and results in a simpler implementation.

The remainder of the paper is organized as follows. In Section II, after a brief introduction to the GSSA methodology, the dynamical model of a DAB converter is presented and its GSSA equivalent model is obtained. The control design is presented in Section III, and Section IV includes an extensive analysis of the ideal sliding dynamics. Then, some simulation results are included in Section $\mathrm{V}$ and, finally, the conclusions are stated in Section VI.

\section{GSSA MODEL OF A DUAL ACTIVE BRIDGE}

\section{A. GSSA methodology}

The GSSA expansion is an averaging technique for power converters (or variable structure systems in general) and aims to capture the fine detail of the state evolution by considering a full Fourier series. Let us define the $k$-th index average (or 


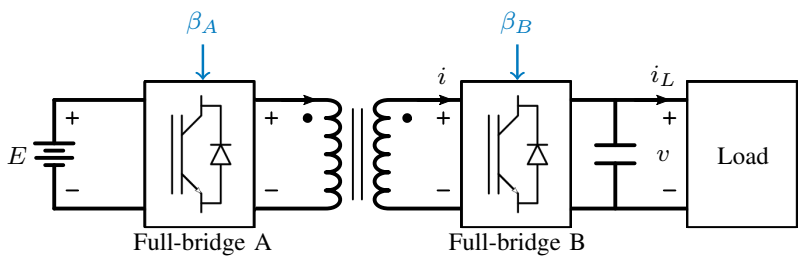

Fig. 1: Dual Active Bridge converter.

the $k$-phasors) as

$$
\langle x\rangle_{k}(t)=\frac{1}{T} \int_{t-T}^{t} x(\tau) e^{-j k \omega \tau} \mathrm{d} \tau,
$$

where $\omega=2 \pi / T$ and $k \in \mathbb{Z}$. Then, a state variable, $x(\tau)$ during the interval $\tau \in[t-T, t]$, can be represented by its Fourier series

$$
x(\tau)=\sum_{k=-\infty}^{+\infty}\langle x\rangle_{k}(t) e^{j k \omega t} .
$$

From [20], the time derivative of the $k$-th coefficient is

$$
\frac{\mathrm{d}}{\mathrm{d} t}\langle x\rangle_{k}=\left\langle\frac{\mathrm{d}}{\mathrm{d} t} x\right\rangle_{k}-j k \omega\langle x\rangle_{k},
$$

and the $k$-th coefficient of the product of two variables, $x(t), y(t)$, is

$$
\langle x y\rangle_{k}=\sum_{l=-\infty}^{+\infty}\langle x\rangle_{k-l}\langle y\rangle_{l} .
$$

For sake of simplicity, when clear from the context, the following notation is used $x_{k}=\langle x\rangle_{k}$.

\section{B. The dual active bridge converter}

Figure 1 shows a simplified scheme of the DAB converter. It consists of a two-port high frequency transformer with a two full-bridge switches connected to each transformer winding and a dc-voltage source, $E$, and a capacitor in the primary and secondary sides, ports $A$ and $B$, respectively.

Neglecting the magnetizing current of the transformer and assuming a transformer ratio of $n=1$, the DAB dynamics can be written as [19]

$$
\begin{aligned}
L \frac{\mathrm{d} i}{\mathrm{~d} t} & =E \beta_{A}-v \beta_{B}-r i \\
C \frac{\mathrm{d} v}{\mathrm{~d} t} & =i \beta_{B}-i_{L},
\end{aligned}
$$

where $i_{L}$ represents a generic load, and control signals $\beta_{A}, \beta_{B}$ are, usually, square wave signals with the form

$$
\begin{aligned}
& \beta_{A}=\operatorname{sign}(\sin (\omega t)) \\
& \beta_{B}=\operatorname{sign}(\sin (\omega t-\delta)) .
\end{aligned}
$$

\section{GSSA model of a DAB converter}

Applying the GSSA transformation to (2a)-(2b)

$$
\begin{aligned}
L \frac{\mathrm{d} i_{1}}{\mathrm{~d} t} & =-j \omega_{s} L i_{1}+E \beta_{A 1}-\left\langle v \beta_{B}\right\rangle_{1}-r i_{1} \\
C \frac{\mathrm{d} v_{0}}{\mathrm{~d} t} & =\left\langle i \beta_{B}\right\rangle_{0}-i_{L 0},
\end{aligned}
$$

where

$$
\begin{aligned}
\left\langle v \beta_{B}\right\rangle_{1} & =v_{1} \beta_{B 0}+v_{0} \beta_{B 1}+v_{2} \bar{\beta}_{B 1}+\ldots \\
\left\langle i \beta_{B}\right\rangle_{0} & =i_{0} \beta_{B 0}+\bar{i}_{1} \beta_{B 1}+i_{1} \bar{\beta}_{B 1}+\ldots
\end{aligned}
$$

and $x_{-k}=\bar{x}_{k}$ has been used. Truncating for $i_{1}, v_{0}$ one gets

$$
\begin{aligned}
L \frac{\mathrm{d} i_{1}}{\mathrm{~d} t} & =-j \omega_{s} L i_{1}+E \beta_{A 1}-v_{0} \beta_{B 1}-r i_{1} \\
C \frac{\mathrm{d} v_{0}}{\mathrm{~d} t} & =i_{0} \beta_{B 0}+\bar{i}_{1} \beta_{B 1}+i_{1} \bar{\beta}_{B 1}-i_{L 0},
\end{aligned}
$$

By using the definition of the average phasors (1), the zero-th and first indices of the control signals in (3a)-(3b) results in

$$
\begin{aligned}
& \beta_{A 0}=0, \quad \beta_{A 1}=-j \frac{2}{\pi} \\
& \beta_{B 0}=0, \quad \beta_{B 1}=-j \frac{2}{\pi} e^{-j \delta},
\end{aligned}
$$

that replaced in $(5 a)-(5 b)$

$$
\begin{aligned}
L \frac{\mathrm{d} i_{1}}{\mathrm{~d} t} & =-\left(r+j \omega_{s} L\right) i_{1}-j \frac{2}{\pi} E+j v_{0} \frac{2}{\pi} e^{-j \delta} \\
C \frac{\mathrm{d} v_{0}}{\mathrm{~d} t} & =-j \frac{2}{\pi}\left(\bar{i}_{1} e^{-j \delta}-i_{1} e^{j \delta}\right)-i_{L 0},
\end{aligned}
$$

resulting in a complex-valued second order nonlinear dynamics. Since $v_{0}, i_{L 0} \in \mathbb{R}$, the Equation (7b) can be written in a more compact way as

$$
C \frac{\mathrm{d} v_{0}}{\mathrm{~d} t}=-I_{1} \frac{4}{\pi} \sin (\theta+\delta)-i_{L 0}
$$

yielding a real-valued nonlinear dynamics where $I_{1}$ and $\theta$ correspond to the modulus and argument of $i_{1}$, respectively, i.e., $i_{1}=I_{1} e^{j \theta}$.

The complex-valued dynamics in (7a) can be written in polar coordinates, separating them into the real and imaginary parts. Then, combining (7a) and (8) the DAB model results in

$$
\begin{aligned}
L \frac{\mathrm{d} I_{1}}{\mathrm{~d} t} & =-r I_{1}-\frac{2}{\pi} E \sin \theta+v_{0} \frac{2}{\pi} \sin (\theta+\delta) \\
\frac{\mathrm{d} \theta}{\mathrm{d} t} & =-\omega_{s}-\frac{2}{\pi L I_{1}} E \cos \theta+v_{0} \frac{2}{\pi L I_{1}} \cos (\theta+\delta) \\
C \frac{\mathrm{d} v_{0}}{\mathrm{~d} t} & =-I_{1} \frac{4}{\pi} \sin (\theta+\delta)-i_{L 0} .
\end{aligned}
$$

Usually, load currents are a mix of resistive and constant power loads (CPLs),

$$
i_{L 0}=\frac{v_{0}}{R_{L}}+\frac{P_{L}}{v_{0}}
$$

where $R_{L}$ and $P_{L}$ are load resistance and the constant power load values, respectively.

\section{SLIDING MODE CONTROLLER}

\section{A. Dynamic extension}

Since the system is non-affine with the control input, a standard sliding mode controller can not be applied. To skip this structural problem, let us extend the dynamics with

$$
\frac{\mathrm{d} \delta}{\mathrm{d} t}=u
$$

where $u$ is the new control input. Then, the overall dynamics is defined by (9) with (11). 


\section{B. Switching manifold}

With the new control definition, the system is relative degree two and requires a switching manifold with a derivative term. The simplest choice is to select a first order dynamics such as

$$
\sigma=\frac{\mathrm{d} v_{0}}{\mathrm{~d} t}+k_{1}\left(v_{0}-v_{0}^{*}\right)
$$

where $k_{1}>0$, defines the time response of the controller and $v_{0}^{*}$ stands for the desired constant voltage value. Notice that assigning this linear dynamics the system response is unequivocally defined by selecting $k_{1}$.

\section{Sliding mode controller}

The sliding mode controller is the control action required to reach and keep on $\sigma=0$. Differentiating (12) with respect to time and using $(9 \mathrm{c})$ and $(11)$ one gets

$$
\frac{\mathrm{d} \sigma}{\mathrm{d} t}=\Psi-\frac{4 I_{1}}{\pi C} \cos (\theta+\delta) u
$$

where, grouping terms,

$$
\begin{aligned}
\Psi= & -\frac{4}{\pi C} \sin (\theta+\delta)\left(k_{1} I_{1}+\frac{\mathrm{d} I_{1}}{\mathrm{~d} t}\right)-\frac{4 I_{1}}{\pi C} \cos (\theta+\delta) \frac{\mathrm{d} \theta}{\mathrm{d} t} \\
& -\frac{1}{C}\left(k_{1} i_{L 0}+\frac{\mathrm{d} i_{L 0}}{\mathrm{~d} t}\right) .
\end{aligned}
$$

The equivalent control, $u_{e q}$, is defined as the control input guaranteeing $\dot{\sigma}=0$. Hence, from (13),

$$
\frac{4 I_{1}}{\pi C} \cos (\theta+\delta) u_{e q}=\Psi .
$$

Using (13) and (15) one can write

$$
\sigma \frac{\mathrm{d} \sigma}{\mathrm{d} t}=\sigma \frac{4 I_{1}}{\pi C} \cos (\theta+\delta)\left(u_{e q}-u\right)
$$

and the control law

$$
u=k \cdot \operatorname{sign}(\sigma \cos (\theta+\delta)),
$$

with $k>\left|u_{e q}\right|$ guarantees $\sigma \dot{\sigma}<0$, and the sliding motion on $\sigma=0$ is ensured.

The knowledge of the sign of $\cos (\theta+\delta)$ in (16) is necessary to ensure the sliding motion. During the numerical simulation stage has been observed that the value of $\cos (\theta+\delta)$ remains positive all time. Additionally, this control action defines an additional (and undesired) sliding surface at $\theta+\delta= \pm \frac{\pi}{2}$. In a practical implementation the following control action will be adopted

$$
u=k \cdot \operatorname{sign}(\sigma) \text {. }
$$

Figure 2 shows the resulting control scheme including: the dynamic extension (11), the switching manifold (12), and the switching control law (17).

\section{IDEAL SLIDING DYNAMICS}

Ideal sliding dynamics occurs when $\dot{\sigma}=\sigma=0$. For an easy analysis, it is assumed a static load composed by a resistor and a CPL, so the current load is assumed with the form (10).

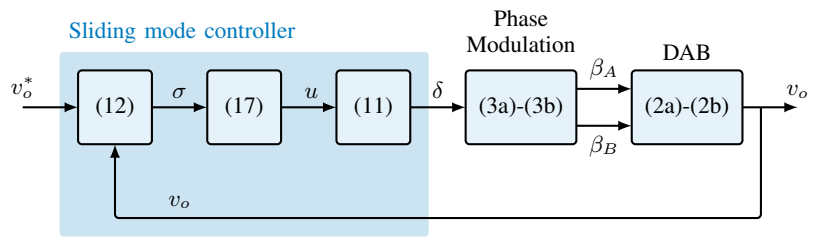

Fig. 2: Control scheme.

\section{A. Voltage dynamics}

From the switching manifold definitions in Section III-B with $\sigma=0$ the voltage dynamics is easily identified as

$$
\frac{v_{0}(s)}{v_{0}^{*}(s)}=\frac{k_{1}}{s+k_{1}},
$$

that corresponds to a first order response with a constant time $\tau=1 / k_{1}$.

\section{B. Remaining sliding dynamics}

On another hand, using $u=u_{e q}$ in (11) and assuming that $v_{0}$ reaches the desired value $v_{0}^{*}$ as shown in Section IV-A, one gets

$$
\frac{\mathrm{d} \delta}{\mathrm{d} t}=\frac{\pi C}{4 I_{1}} \frac{\Psi}{\cos (\theta+\delta)} .
$$

Replacing $\Psi$ from (14), using (10) and after some algebra,

$$
\begin{aligned}
I_{1} \cos (\theta+\delta)\left(\frac{\mathrm{d} \theta}{\mathrm{d} t}+\frac{\mathrm{d} \delta}{\mathrm{d} t}\right) & =-\sin (\theta+\delta)\left(k_{1} I_{1}+\frac{\mathrm{d} I_{1}}{\mathrm{~d} t}\right) \\
& -k_{1} \frac{\pi}{4}\left(\frac{v_{0}^{*}}{R_{L}}+\frac{P_{L}}{v_{0}^{*}}\right) .
\end{aligned}
$$

Let us define the auxiliary variable

$$
z=I_{1} \sin (\theta+\delta)
$$

Differentiating with respect to the time (19) together with (18), the new variable $z$ exhibits a first order dynamics

$$
\frac{\mathrm{d} z}{\mathrm{~d} t}=-k_{1} z-k_{1} \frac{\pi}{4}\left(\frac{v_{0}^{*}}{R_{L}}+\frac{P_{L}}{v_{0}^{*}}\right),
$$

that is stable since $k_{1}>0$, and stabilises at

$$
z^{*}=-\frac{\pi}{4}\left(\frac{v_{0}^{*}}{R_{L}}+\frac{P_{L}}{v_{0}^{*}}\right) .
$$

From (20), one knows that $z$ asymptotically tends to $z^{*}$, thus implying that the ideal sliding dynamics converge to the manifold defined by

$$
z^{*}=I_{1} \sin (\theta+\delta) .
$$

Assuming that (22) is reached, and replacing it into the dynamics $(9 a)-(9 b)$ one gets

$$
\begin{aligned}
L \frac{\mathrm{d} I_{1}}{\mathrm{~d} t} & =-r I_{1}-\frac{2}{\pi} E \sin \theta+v_{0}^{*} \frac{2}{\pi I_{1}} z^{*} \\
\frac{\mathrm{d} \theta}{\mathrm{d} t} & =-\omega_{s}-\frac{2}{\pi L I_{1}} E \cos \theta+v_{0}^{*} \frac{2}{\pi L I_{1}^{2}} \sqrt{I_{1}^{2}-z^{* 2}} .
\end{aligned}
$$

Since this system is highly non-linear, the stability of the small-signal model around an equilibrium point will 
be analyzed. After some algebra, the equilibrium points, denoted by $I_{1}^{*}, \theta^{*}$ are the solutions of

$$
E^{2}-v_{0}^{* 2}+v_{0}^{*} z^{*} r=\frac{\pi}{4}\left(r^{2}+\omega_{s}^{2} L^{2}\right) I_{1}^{* 2}-v_{0}^{*} \omega_{s} L \sqrt{I_{1}^{* 2}-z^{* 2}},
$$

and

$$
\tan \theta^{*}=\frac{-\pi r I_{1}^{* 2}+2 v_{0}^{*} z^{*}}{-\pi \omega_{s} L I_{1}^{* 2}+2 v_{0}^{*} \sqrt{I_{1}^{* 2}-z^{* 2}}} .
$$

Notice that the quadratic function (24) have four possible solutions, but only positive values are admissible since $I_{1}$ is the modulus of $i_{1}$. On another hand, from (23b), periodic solutions for $\theta^{*}$ are obtained.

The Jacobian of (23), evaluated at the equilibria yields

$$
J_{\mathrm{ISD}}=\left(\begin{array}{cc}
-\frac{r}{L}-\frac{2 v_{0}^{*} z^{*}}{\pi I^{* 2} L} & \omega_{s} I_{1}^{*}-\frac{2 v_{o}^{*} \sqrt{I_{1}^{* 2}-z^{* 2}}}{\pi L I_{1}^{*}} \\
\frac{\omega_{s}}{I_{1}^{*}}-\frac{2 v_{0}^{*} z^{* 2}}{\pi I_{1}^{* 3} L \sqrt{I_{1}^{* 2}-z^{* 2}}} & -\frac{r}{L}+\frac{2 v_{0}^{*} z^{*}}{\pi I_{1}^{* 2} L}
\end{array}\right) .
$$

The equilibrium point is locally stable if, and only if, all the coefficients of the characteristic polynomial of $J_{\text {ISD, }}$,

$$
\lambda^{2}-\operatorname{tr}\left(J_{\mathrm{ISD}}\right) \lambda+\operatorname{det}\left(J_{\mathrm{ISD}}\right),
$$

have the same sign. Then, the two necessary and sufficient conditions to assure stability around the equilibrium point are $\operatorname{tr}\left(J_{\text {ISD }}\right)<0$ and $\operatorname{det}\left(J_{\text {ISD }}\right)>0$,

$$
\begin{aligned}
\operatorname{tr}\left(J_{\text {ISD }}\right) & =-2 \frac{r}{L}<0 \\
\operatorname{det}\left(J_{\text {ISD }}\right) & =\omega_{s}^{2}+\frac{r^{2}}{L^{2}}-\frac{2 v_{0}^{*} \omega_{s}}{\pi L \sqrt{I_{1}^{* 2}-z^{* 2}}}>0 .
\end{aligned}
$$

The first condition is automatically achieved, since the second condition defines a range of admissible values. Notice that the stability does not depends on the control gains.

A numerical example of the obtained analysis is carried out using the parameters of the DAB converter simulated in Section V. With a load values $R_{L}=100 \Omega$ and $P_{L}=100 \mathrm{~W}$, the auxiliary value (21)

$$
z^{*}=-2.277,
$$

that, from (24) results in two possible current values

$$
\begin{aligned}
& I_{1}^{*}=2.282 \mathrm{~A} \\
& I_{1}^{*}=40.452 \mathrm{~A},
\end{aligned}
$$

with determinant values

$$
\begin{aligned}
& \operatorname{det}\left(J_{\text {ISD }}\right)=-3.563 \cdot 10^{12} \\
& \operatorname{det}\left(J_{\text {ISD }}\right)=1.229 \cdot 10^{10}
\end{aligned}
$$

concluding that there exists two possible values for $I_{1}^{*}$, but only $I_{1}^{*}=40.452 \mathrm{~A}$ is stable. With this current value in (25)

$$
\theta^{*}=-3.076+2 n \pi, \quad n \in \mathbb{Z},
$$

and using (19)

$$
\delta^{*}=3.0194+2 n \pi, \quad n \in \mathbb{Z} .
$$

Figure 3 shows the trajectories of (23) with the parameters in Section $\mathrm{V}$ and $R_{L}=100 \Omega$ and $P_{L}=100 \mathrm{~W}$, for

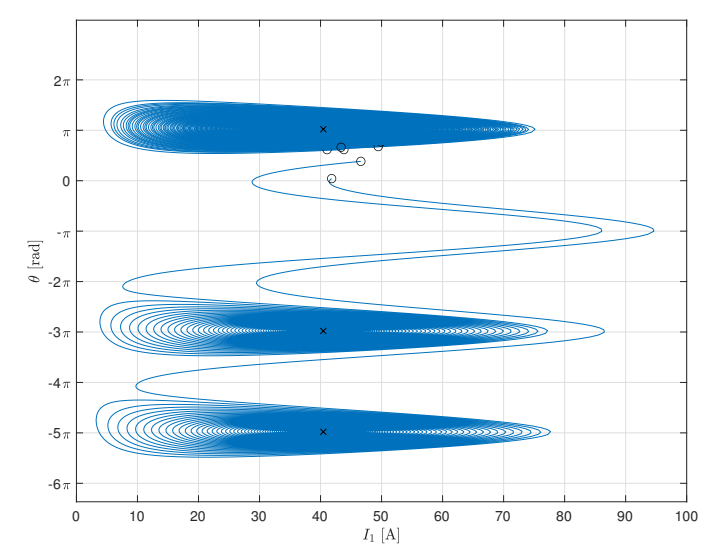

Fig. 3: Simulation results: ideal sliding dynamics in (23) for a batch of initial conditions. Initial conditions are identified with a circle, and equilibrium points with a cross.

different initial conditions. It can be observed how trajectories stabilize at different equilibrium points with values $\left(I_{1}^{*}, \theta^{*}\right)=(40.452,-3.076+2 n \pi)$. The definition of the region of attraction, if possible, is a more complicated task and is left for future works.

\section{Simulations Results}

Some numerical simulations using Matlab-Simulink have been carried out to test the proposed controller. The parameters of the DAB converter were: $C=1500 \mu \mathrm{F}, L=8 \mu \mathrm{H}$, $r=0.006 \Omega, E=40 \mathrm{~V}$, and the switching frequency was $f=25 \mathrm{kHz}$ (then $\omega_{s}=2 \pi f$ ).

The gain of the sliding mode controller, in (17), was set to $k=10^{3}$. The switching manifold in (12) is defined with $k_{1}=2000$ that corresponds to a settling time of $t_{s}=2 \mathrm{~ms}$. The simulation has been run at a fixed step size of $5 \cdot 10^{-8} \mathrm{~s}$ with the ode4 (Runge-Kutta) solver.

\section{A. Simulations with the GSSA model}

As a first stage, the controller has been tested using the GSSA model in (9). The desired voltage value was set to $v_{0}^{*}=40 \mathrm{~V}$, the load values were $R_{L}=100 \Omega$ and $P_{L}=$ $100 \mathrm{~W}$. The voltage initial condition was $v_{0}(0)=35 \mathrm{~V}$.

Figure 4 (bottom) shows that the sliding motion is reached after, approximately, $0.5 \mathrm{~ms}$ and, consequently, the output voltage is regulated after $2 \mathrm{~ms}$, following the design requirements. The simulation results shown in Figure 5 (top) also show that the value of $\cos (\theta+\delta)$ remains positive and close one. This confimrs removing that term in (16), so that, for implementation purposes, the controller is turns to an output feedback scheme. Finally, as expected, the equilibrium value for the current variables, $I_{1}$ and $\theta$, are the ones obtained in the numerical analysis of the ideal sliding dynamics in Section IV.

\section{B. Realistic simulations}

In a second stage, the controller has been simulated using the model (2), which implies that the control signals follow the waveforms in (3). Additionally, since $f=25 \mathrm{kHz}$, 

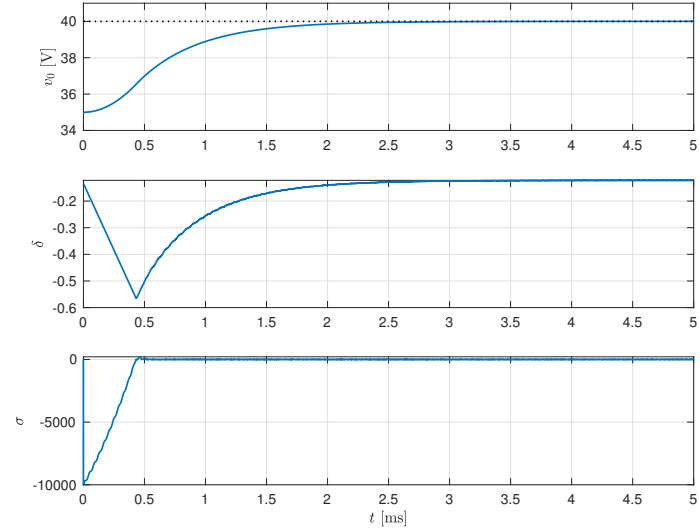

Fig. 4: Simulation results with the GSSA model in (9): (top) the output voltage, $v_{0}$, (mid) the phase shift, $\delta$ and (bottom) the switching manifold, $\sigma$.
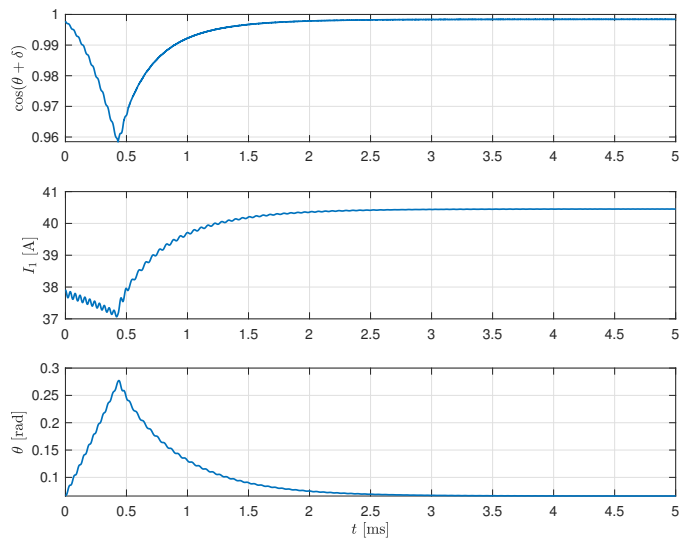

Fig. 5: Simulation results with the GSSA model in (9): (top) the value of $\cos (\theta+\delta)$, (mid) the current modulus, $I_{1}$ and (bottom) the current argument, $\theta$

the variable, $\delta$, is sampled with $T=40 \mathrm{~ns}$. With the same control parameters from the previous Section, the test consists in changing the voltage reference and the load values as follows:

$$
\begin{aligned}
v_{0}^{*} & = \begin{cases}39 \mathrm{~V} & t<5 \mathrm{~ms} \\
40 \mathrm{~V} & t \geq 5 \mathrm{~ms}\end{cases} \\
R_{L} & = \begin{cases}100 \Omega & t<20 \mathrm{~ms} \\
6 \Omega & t \geq 20 \mathrm{~ms}\end{cases} \\
P_{L} & = \begin{cases}0 \mathrm{~W} & t<10 \mathrm{~ms} \\
100 \mathrm{~W} & 10 \leq t<15 \mathrm{~ms} \\
200 \mathrm{~W} & t \geq 15 \mathrm{~ms}\end{cases}
\end{aligned}
$$

Figure 6 shows the output voltage, the phase shift control angle and the switching manifold. The voltage is regulated at the desired value when changing the reference value and in face of load changes, with the settling time of $2 \mathrm{~ms}$. With respect to the previous simulations, high-frequency oscillations appear because of the switching signals of $\beta_{A}$ and $\beta_{B}$. The calculated GSSA variables are shown in Figure 7. On top, the extracted GSSA output voltage shows a
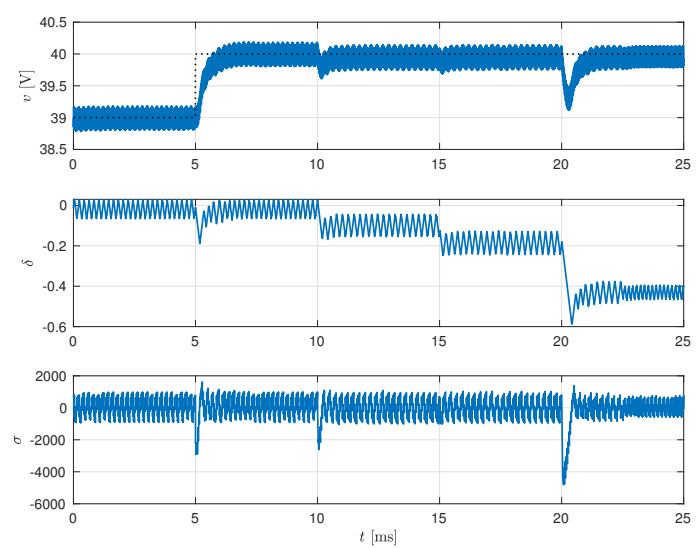

Fig. 6: Simulation results with the original model in (2): (top) the output voltage, $v$, (mid) the phase shift, $\delta$ and (bottom) the switching manifold, $\sigma$.
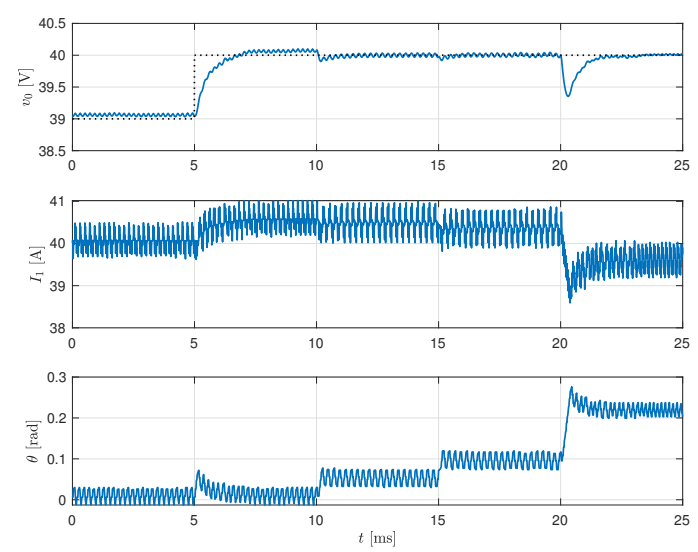

Fig. 7: Simulation results with the original model in (2): (top) the output GSSA voltage, $v_{0}$, in red, (mid) the modulus of the GSSA current, $I_{1}$ and (bottom) the argument of the GSSA current, $\theta$.

small steady state error, that is associated to all disregarded harmonics. The GSSA current, in polar coordinates, is shown in the mid and bottom plots.

\section{CONCLUSIONS}

A sliding based control algorithm is proposed for a DAB. The control design is based on the GSSA approximation and includes a dynamics extension to solve the problem of having the input with a non-affine form.

Thanks to the sliding motion, the obtained controller allows to freely design the output dynamics, independently of the load changes. Additionally, it has been observed that the dependence on the term $\cos (\theta+\delta)$ can be removed, resulting in an output feedback scheme (compared with the state feedback algorithm in [17]). In overall, the controller offers good performance and robustness results .

Future works include the implementation of the controller in a real plant.

\section{REFERENCES}

[1] R. W. A. A. De Doncker, D. M. Divan, and M. H. Kheraluwala, "A three-phase soft-switched high-power-density dc/dc converter for high- 
power applications," IEEE Trans. on Industry Applications, vol. 27, no. 1, pp. 63-73, 1991.

[2] R. K. Behera and O. Ojo, "Modeling and control of DAB converter for solar micro-grid application," in Proc. 6th International Conference on Power Electronics Systems and Applications, 2015.

[3] B. Zhao, Q. Yu, and W. Sun, "Extended-phase-shift control of isolated bidirectional DC-DC converter for power distribution in microgrid," IEEE Trans. on Power Electronics, vol. 27, no. 11, pp. 4667-4680, 2012.

[4] F. Esteban, F. Serra, and C. De Angelo, "Control of a DC-DC dual active bridge converter in DC microgrids applications," IEEE Latin America Transactions, Early Access.

[5] Q. Xiao, L. Chen, H. Jia, P. W. Wheeler, and T. Dragincevina, "Model predictive control for dual active bridge in naval DC microgrids supplying pulsed power loads featuring fast transition and online transformer current minimization," IEEE Trans. on Industrial Electronics, vol. 67 , no. 6, pp. 5197-5203, 2020.

[6] F. Krismer and J. W. Kolar, "Accurate small-signal model for the digital control of an automotive bidirectional dual active bridge," IEEE Trans. on Power Electronics, vol. 24, no. 12, pp. 2756-2768, 2009.

[7] S. Inoue and H. Akagi, "A bidirectional DC-DC converter for an energy storage system with galvanic isolation," IEEE Trans. on Power Electronics, vol. 22, no. 6, pp. 2299-2306, 2007.

[8] H. Qin and J. W. Kimball, "Closed-loop control of DC-DC dualactive-bridge converters driving single-phase inverters," IEEE Trans. on Power Electronics, vol. 29, no. 2, pp. 1006-1017, 2014.

[9] R. Griñó and A. Dòria-Cerezo, "Modelling and simulation of a magnetically coupled multiport dc-dc converter," in Proc. 24th Int. Conf. on Emerging Technologies and Factory Automation, 2019.

[10] B. P. Baddipadiga and M. Ferdowsi, "Dual loop control for eliminating dc-bias in a dc-dc dual active bridge converter," in Proc. 3rd International Conference on Renewable Energy Research and Applications, 2014.

[11] D. Das, S. Mishra, and B. Singh, "Design architecture for continuous time control of dual active bridge converter," IEEE Journal of Emerging and Selected Topics in Power Electronics, Early Access.

[12] D.-D. Nguyen, D.-H. Nguyen, M. Ta, and G. Fujita, "Sensorless feedforward current control of dual-active-bridge dc/dc converter for micro-grid applications," IFAC-PapersOnLine, vol. 51, no. 28, pp. 333-338, 2018.

[13] I. Askarian, S. Bagawade, M. Pahlevani, A. M. Knight, and A. Bakhshai, "Robust digital nonlinear control system for dual active bridge (DAB) dc/dc converters with asymmetric half-cycle modulation," IEEE Journal of Emerging and Selected Topics in Industrial Electronics, vol. 1, no. 2, pp. 123-132, 2020.

[14] M. Cupelli, S. K. Gurumurthy, S. K. Bhanderi, Z. Yang, P. Joebges, A. Monti, and R. W. De Doncker, "Port controlled Hamiltonian modeling and IDA-PBC control of dual active bridge converters for dc microgrids," IEEE Trans. on Industrial Electronics, vol. 66, no. 11, pp. 9065-9074, 2019.

[15] R. V. Meshram, M. Bhagwat, S. Khade, S. R. Wagh, A. M. Stankovic, and N. M. Singh, "Port-controlled phasor Hamiltonian modeling and IDA-PBC control of solid-state transformer," IEEE Trans. on Control Systems Technology, vol. 27, no. 1, pp. 161-173, 2019.

[16] E. L. S. da Silva, A. L. Kirsten, and D. J. Pagano, "Discrete sps control of a dab converter using partial feedback linearization," in Proc. IEEE 15th Brazilian Power Electronics Conference and 5th IEEE Southern Power Electronics Conference, 2019.

[17] Y. Jeung and D. Lee, "Voltage and current regulations of bidirectional isolated dual-active-bridge dc-dc converters based on a double-integral sliding mode control," IEEE Trans. on Power Electronics, vol. 34, no. 7, pp. 6937-6946, 2019.

[18] H. Qin and J. W. Kimball, "Generalized average modeling of dual active bridge dc-dc converter," IEEE Trans. on Power Electronics, vol. 27, no. 4, pp. 2078-2084, 2012.

[19] J. A. Mueller and J. W. Kimball, "An improved generalized average model of dc-dc dual active bridge converters," IEEE Trans. on Power Electronics, vol. 33, no. 11, pp. 9975-9988, 2018.

[20] S. R. Sanders, J. M. Noworolski, X. Z. Liu, and G. C. Verghese, "Generalized averaging method for power conversion circuits," IEEE Trans. on Power Electronics, vol. 6, no. 2, pp. 251-259, 1991.

[21] C. Batlle, E. Fossas, R. Griñó, and S. Martínez, "Generalized state space averaging for port controlled hamiltonian systems," in Proc. IFAC World Congress, 2005.
[22] C. Gaviria, E. Fossas, and R. Griñó, "Robust controller for a fullbridge rectifier using the IDA approach and GSSA modeling," IEEE Trans. on Circuits and Systems I, vol. 52, no. 3, pp. 609-616, 2005.

[23] C. Batlle, A. Dòria-Cerezo, and E. Fossas, "Bidirectional power flow control of a power converter using passive Hamiltonian techniques," Int. Journal of Circuit Theory and Applications, vol. 36, no. 7, pp. 769-788, 2008.

[24] S. Bacha, I. Munteanu, and A. I. Bractu, Power Electronic Converters Modeling and Control. Springer-Verlag, 2014.

[25] T. L. Nguyen, G. Griepentrog, and V. T. Phung, "Modeling and control of dual active bridge converter with two control loops and output filter," in Proc. 43th Annual Conf. of the IEEE Industrial Electronics Society, 2017.

[26] M. Cupelli, S. K. Gurumurthy, and A. Monti, "Modelling and control of single phase DAB based MVDC shipboard power system," in Proc. 43th Annual Conf. of the IEEE Industrial Electronics Society, 2017.

[27] S. K. Gurumurthy, M. Cupelli, and A. Monti, "State space modelling and control of triple phase shift modulated single phase DAB for shipboard power system," in Proc. 43th Annual Conf. of the IEEE Industrial Electronics Society, 2017. 